\title{
The inner representation of the external world - from conditioned reflexes to high level mental functions in the light of Nobel Prizes
}

\author{
Szilágyi T, Orbán-Kis K \\ Department of Physiology, University of Medicine and Pharmacy of Târgu Mures, Romania
}

In this paper the seminal results of the 2014 Nobel Prize in Physiology or Medicine Laureates are presented. First, a historical review of the development of our knowledge is provided along with the major paradigm shifts, by looking at the Nobel prizes awarded in the field of neuroscience in the last 110 years. We outline the major discoveries that were necessary for humankind to pass through the road leading to the remarkable understanding of high level mental functions, which led to this year's Nobel Prize award. Next, the ground breaking discoveries of this year Nobel laureates are presented, which provide insights how neural representations of the environment are formed in the association cortices. These cortical areas are many synapses away from sensory receptors and motor outputs, and their activity do not reflect directly the activation patterns of the receptor population, but depends more strongly on intrinsic cortical computations. We also present how ensembles of specialized cells work together to compute complex cognitive functions and behaviour.

Keywords: Nobel Prize, neurosciences, positioning system, higher cognitive functions, cell ensembles.

Received: 21 November 2014 / Accepted: 01 December 2014

On 6 October 2014 it was announced that the Nobel Prize in Physiology or Medicine 2014 was divided, one half awarded to John O'Keefe, the other half jointly to MayBritt Moser and Edvard I. Moser "for their discoveries of cells that constitute a positioning system in the brain" [1].

Animals and humans respond amazingly fast and precisely to changes in their environment. For the current reactions and decisions they also use their past experience. By these reactions living organisms achieve an optimal interaction with their environment. A fundamental question is: how the information from the environment is encoded, stored and retrieved in the nervous system, and finally how this neural code is used to give rise to proper reactions.

Deciphering the morphological basis and physiological processes which enable the powerful information processing in the nervous system has been in the focus of interest of millions of scientists in the last century. Bearing in mind that the field of neurosciences is extremely complex, and to summarize only the most important discoveries would need thousands of pages, in this paper we will try to synthesize the development of our knowledge, the major paradigm shifts, by looking at the Nobel prizes awarded in this field. In this way we will try to outline the major discoveries which were necessary for humankind to pass through the road leading to the remarkable understanding of high level mental functions, which were awarded by Nobel Prize this year.

* Correspondence to: Tibor Szilágyi

E-mail: szilagyi@umftgm.ro
110 years ago, in his presentation speech on December 10, 1904 Professor the Count K.A.H. Mörner, Rector of the Royal Caroline Institute stated that "The aim of science is the acquisition of knowledge, the value of which should not be measured by the ease with which it can be brought immediately into practical usefulness. ... The questions of the investigator to Nature and the yearning which fires his desire are directed in the first instance to the gaining of new and deeper knowledge. Other factors also show that Nobel greatly appreciated investigators who were seeking knowledge for its own sake without considering other circumstances." [2]. Today, more than a century later, when applied research is the most widely accepted, this idea should be thought-provoking.

In 1904 the Nobel prize was awarded to Ivan Petrovich Pavlov "in recognition of his work on the physiology of digestion, through which knowledge on vital aspects of the subject has been transformed and enlarged" [3]. Pavlov, however, made his mark as a scholar not only in the field of digestive physiology, but he was a pioneer of chronic experiments, which were designed to understand the normal functions of animals by observing their long-term physiological processes. These experiments allowed the discovery of conditioned reflexes, which made possible to study psychic activity objectively. Subjective methods were replaced by experimental ones investigating the very complex interrelations between an organism and its external environment. [2]

Two years later, in 1906, the Nobel Prize in Physiology or Medicine was awarded jointly to Camillo Golgi and 
Santiago Ramón y Cajal "in recognition of their work on the structure of the nervous system" [4]. Obtaining a clear insight into the structure and functioning of the nervous system, "the material foundation of mental life"[5], has been associated with great difficulties. Golgi's new staining method, which impregnates a limited number of neurons at random, permitted for the first time a clear visualization of a nerve cell body with all its processes in its entirety. However, Golgi believed that the observations of ramified nerve fibers supports the 'reticular theory', which considered the nervous system a syncytial system, consisting of nervous fibers forming an intricate network, and that the nervous impulse propagated along this diffuse network. On the contrary, Cajal supported the idea that the nervous system is made up of billions of separate nerve cells. This is the modern basic principle of the organization of the nervous system. Cajal's opus "Textura del Sistema Nervioso del Hombre y los Vertebrados" (1894-1904) provided the foundation of modern neuroanatomy, with a detailed description of nerve cell organization in the central and peripheral nervous system of many different animal species.

The Nobel Prize in Physiology or Medicine 1932 was awarded jointly to Sir Charles Scott Sherrington and Edgar Douglas Adrian "for their discoveries regarding the functions of neurons" [6].

Sherrington provided the basis of understanding how the nervous system receives, controls, utilizes, and responds to information from the external world. He established general laws for the origin and cooperation of the reflexes in the organism. He demonstrated that reflexes are not acting independently from each other, but the motor response is the result of confluence of reflex arcs from many different sensory inputs onto one output neuron. The foundation of neuronal information integration was laid down. "The neurone has the capacity of to a certain extent gathering up and summing these different, simultaneous or rapidly succeeding impulses; the inhibiting and stimulating forces can then wholly or partly counterbalance each other, and the result will be decided by which of them obtains the upper hand for the time being. Both are equally necessary for the normal course of the reflexes and they must cooperate intimately." [5] Sherrington introduced the term 'synapse' for the gap that Cajal postulated between nerve cells.

Adrian used radio amplifiers, by means of which he could record biopotentials of a few microvolts. By ingenious technical artifices, electrical impulses generated by single nerve fibers could be recorded. Today we call these impulses action potentials, whose fundamental properties were summarized in the award ceremony speech held by Liljestrand: "The light which falls on the retina of the eye, the slight contact of the skin, or the factors which cause pain in a wound, all exercise their influence, as Adrian has shown, by giving rise to impulses of fundamentally the same kind in the nerve fibers by the mediation of the special sense organs." It was revealed that the signals are the same also in sensory and motor fibers. The "all or none" nature of nerve impulses was demonstrated, and that a stronger stimulus does not evoke larger impulses but a more rapid stream of impulses, and can activate more single nerve fibers. By these observations the basic encoding scheme used by the nervous system was deciphered. It was discovered what we now call the frequency code and population code.

The Nobel Prize in Physiology or Medicine 1936 was awarded jointly to Sir Henry Hallett Dale and Otto Loewi "for their discoveries relating to chemical transmission of nerve impulses" [7]. Once it was firmly established that the nervous system is composed from intricately interconnected individual nerve cells, which communicate through tiny contacts, the synapses, the next question was raised: how is the signal transmitted across the gaps that separate the cells. At that time opinions were divided between scientist who believed that the message was electrical and those who argued that chemicals must be involved. Working on isolated heart preparations, Otto Loewi very elegantly proved that chemicals act as the messenger. By stimulating the vagus nerve fibers he could demonstrate the release of a substance, what he called vagusstoff. Dale discovered that acetylcholine and the vagusstoff are one and the same substance, and that acetylcholine is produced naturally in the body. Dale and his pupils proved that acetylcholine acts on many tissues and organs other than the heart, affects the activity of ganglia of the autonomic nervous system, and it plays a role in the production of muscular contractions. It was also demonstrated that acetylcholine released from nerve endings is almost immediately destroyed once it has carried out its task.

The Nobel Prize in Physiology or Medicine 1944 was awarded jointly to Joseph Erlanger and Herbert Spencer Gasser "for their discoveries relating to the highly differentiated functions of single nerve fibres" [8]. They have shown that nerve fibers conduct impulses with different speed depending on their thickness. On this basis, fibers were classified into three distinct types, and it was also shown that each type requires different stimulus intensity to obtain a response. Erlanger and Gasser concluded that different fibers transmit different kinds of impulses, e.g. the perception of pain is largely mediated by very thin, slowly conducting fibers, whereas touch and regulation of muscle activity is mediated by rapidly conducting fibers.

Recognizing the importance of differences in conduction speed was a forerunner for understanding a new dimension of coding: the exact timing of impulses. In his award presentation speech Ragnar Granit (Nobel laureate himself later, in 1967) made the following statement: "In the brain and the spinal cord the time ratios of the impulses are of primary importance for the cooperation of the nerve cells. A difference of 0.001-0.005 seconds in the time of arrival of impulses means that a given path may be found opened or closed for their passage onwards" [9].

The Nobel Prize in Physiology or Medicine 1963 was awarded jointly to Sir John Carew Eccles, Alan Lloyd Hodgkin and Andrew Fielding Huxley "for their discov- 
eries concerning the ionic mechanisms involved in excitation and inhibition in the peripheral and central portions of the nerve cell membrane" [10]. The discovery of Hodgkin and Huxley is a milestone on the road towards the understanding of the nature of excitability. They have demonstrated that the rising phase of the action potentials corresponded to an increase in sodium permeability of the cell membrane, which after about half a millisecond, in the falling phase, is replaced by an increase in potassium permeability. Both phases were measured quantitatively and a mathematical model was fitted to the data. Their model could predict correctly the fundamental attributes of excitability. This model, the so called Hodkin-Huxley equations, forms even today the basis of realistic computer modeling of electrical activity of nerve cells.

Eccles has shown that excitation and inhibition are expressed by changes of membrane potential (in modern terminology the postsynaptic potentials). There are two kinds of synapses, one excitatory, the other inhibitory. Eccles demonstrated that if the arriving impulse is connected to excitatory synapses, the membrane potential decreases, and if a threshold value is reached, the cell fires of an impulse. If the arriving impulse is connected to inhibitory synapses, the membrane potential increases and, as a consequence, the impulse discharge is inhibited. Thus excitation and inhibition correspond to ionic currents which push the membrane potential in opposite directions.

The Nobel Prize in Physiology or Medicine $\mathbf{1 9 7 0}$ was awarded jointly to Sir Bernard Katz, Ulf von Euler and Julius Axelrod "for their discoveries concerning the humoral transmitters in the nerve terminals and the mechanism for their storage, release and inactivation" [11].

Dale and Loewi already showed earlier that impulse transmission between neurons takes place by chemical means. The discoveries awarded in 1970 greatly refined our understanding of synaptic transmission. Katz demonstrated that acetylcholine, the messenger substance between the motor nerve and the muscle end-plate, is stored in synaptic vesicles, and it is released from the nerve terminals in small quanta under the influence of calcium. Whether the quantal release corresponds to the liberation of neurotransmitter from a single synaptic vesicle or other molecular mechanisms are involved is a matter of debate even today. Ulf von Euler showed that noradrenaline is stored and synthesized within the nerve fibers themselves. Axelrod discovered that, unlike acetylcholine, which is inactivated by enzymes, much of the noradrenaline after it has finished transmitting the nerve impulse, is taken up back into the presynaptic terminals (neurotransmitter re-uptake).

Knowledge of these subcellular details of synaptic transmission stimulated research for fundamentally new pharmacological treatment methods of mental diseases and psychical disturbances.

The Nobel Prize in Physiology or Medicine 1981 was divided, one half awarded to Roger W. Sperry "for his discoveries concerning the functional specialization of the cerebral hemispheres", the other half jointly to David H. Hubel and Torsten N. Wiesel "for their discoveries concerning information processing in the visual system"[12].

In human patients whose cerebral hemispheres had been surgically separated in order to control otherwise intractable epilepsy, Sperry was able to show that a conscious mind exists in each hemisphere, and they are highly specialized. The isolated left hemisphere is concerned with abstract thinking, symbolic relationships and logical analysis of details, particularly temporal relationships. It is indispensable for mathematical calculations, speech and writing. It works more computer-like and it is dominant in all activities concerning the relations with the external world. The right hemisphere lacks almost entirely the ability to calculate, and generally lacks the possibility to communicate with the outside world, but is clearly superior in space perception tasks, comprehension of complex relationships, face recognition, interpretation of auditory impressions and in comprehension of music. The right hemisphere is more involved in the emotional context of behavior. The famous words of Sperry highlight these differences: "The great pleasure and feeling in my right brain is more than my left brain can find the words to tell you."

These result facilitated progress regarding the mindbrain problem too; a revised concept of the nature of consciousness and its fundamental relation to brain functions has emerged.

To quote from the award ceremony speech, "Hubel and Wiesel have succeeded in breaking the code of the message which the eyes send to the brain and have thereby given us insight into the neuronal processes underlying our visual experiences" [13]. They demonstrated that the retinal image is not simply transmitted point by point to visual centers in the brain, but the visual information undergoes a step-wise analysis, and in the visual cortex each cell has its specific function and is responsible for a specific detail in the pattern of the retinal image. The cortical cells, which are arranged in an orderly manner in columns, interpret the picture projected to the retina in respect to contrast, linear patterns and movement. The cells within each column are sensitive to the same particular detail of a small region of the retinal picture.

The Nobel Prize in Physiology or Medicine 1991 was awarded jointly to Erwin Neher and Bert Sakmann "for their discoveries concerning the function of single ion channels in cells" [14].

It has long been known that excitability depends on changes of the membrane potential, and these changes are brought about by transmembrane ionic currents. Hodgkin and Huxley characterized and quantitatively analyzed these currents already in 1952. It has also long been speculated that membrane currents are flowing through specific ion channels, but Neher and Sakmann were the first to show that these channels actually exist. They developed a technique (the patch-clamp) by which the extremely small currents, flowing through a single ion channel molecule, could 
be measured. By this method it was possible to measure exactly when a single ion channel is opened or closed, that is when a single molecule changes its conformation, which is a totally unique level of resolution. This technique is used even today in thousands of laboratories across the world.

Combining the patch-clamp technique with new methods through which different parts of the ion channel molecules could be modified, it became possible to elucidate the functional role of the different parts of the molecule, e.g. what makes an ion channel selective to a certain type of ion, how can it sense the membrane potential or be sensitive to a particular signaling molecule.

Ion channels are found in the membrane of all living cells. Several diseases are caused or influenced by a modified ion channel function. Today a continuously increasing number of congenital or acquired channelopathies are discovered. Many drugs act on specific types of ion channel. Understanding their mechanism of action is of paramount importance for improving therapeutic methods. With the help of the technique of Neher and Sakmann it is now possible to design drugs with optimal effect on particular ion channels involved in a given disease.

The Nobel Prize in Physiology or Medicine 2000 was awarded jointly to Arvid Carlsson, Paul Greengard and Eric R. Kandel "for their discoveries concerning signal transduction in the nervous system" [15]. They have made important discoveries regarding slow synaptic transmission and neuronal plasticity.

Carlsson discovered that dopamine is a true neurotransmitter in the brain, not a mere precursor of noradrenaline. $\mathrm{He}$ demonstrated that the striatal dopmaninergic system has great importance in the control of movements. His research led to the realization that Parkinson's disease is caused by a lack of dopamine in this part of the brain, and that the dopamine precursor L-dopa is an efficient drug to treat Parkinson's disease. He also provided evidence that perturbed dopamine regulation is associated with some forms of mental illness, e.g. schizophrenia, and demonstrated the mode of action of drugs used for the treatment of schizophrenia.

Greengard explored the chain of events taking place after dopamine binds to its receptor in the cell membrane. Activation of dopamine receptors $\left(D_{1}\right)$ causes an elevation of cyclic AMP, which in turn activates protein kinase $\mathrm{A}$, which phosphorylates other target proteins with different functions in the nerve cell. When ion channels are phosphorylated, the excitability of the nerve cell changes. Dopamine can influence a key regulatory protein, DARPP-32 (dopamine and cAMP regulated phosphoprotein of $32 \mathrm{kDa}$ ), which indirectly changes the function of a large number of other proteins. The resulting change in the function of the neurons may last from seconds to hours.

Kandel has demonstrated that changes in synaptic function are central for learning and memory. He showed that short term memory, which lasts from minutes to hours, is based on the amplification of synaptic transmission caused by phosphorylation of certain ion channel proteins, that is by the mechanism described by Greengard. During the formation of long term memory, which last for weeks, a change in protein synthesis occurs. Consequently the shape of the synapse can change and its size can increase, thereby it can induce a long lasting increase of synaptic function. Kandel's initial experiments were carried out on sea slugs, but later he was able to show that the same type of long term changes of synaptic function applies to mammals too. The fundamental mechanisms that he has discovered are also applicable to humans.

After reviewing the awarded discoveries which were prerequisite to this year Nobel Prize, we will present in more detail the Nobel Prize in Physiology or Medicine 2014 which was awarded to John O'Keefe, May-Britt Moser and Edvard I. Moser "for their discoveries of cells that constitute a positioning system in the brain" [1].

The ability of the nervous system to adapt decision making and behaviour according to changes in the internal and external environment requires recreation of the properties of the environment in activity patterns of the neurons. Dynamic clusters of cells with activity pattern correlated with features of the external world can form neural representations of the environment [16]. Representations are formed in a hierarchical way. At the bottom of the hierarchy are the earliest stages of sensory systems, where sensory maps reproduce the spatial organization of the sensory receptors [17]. This level of neural representations, especially for the visual system, is relatively well understood. Representations at higher levels, in the association cortices, do not reflect directly the activation patterns of the receptor population, but depends more strongly on intrinsic cortical computations. Much less is known about these levels, which are many synapses away from sensory receptors and motor outputs, and form the basis for complex cognitive functions and behaviour. The ground breaking discoveries of this year Nobel laureates provide insights to this level of mental functions.

There are nerve cells in the medial entorhinal cortex and the hippocampus that enable a sense of place and navigation. These cells form multiple maps that are needed for recognizing and remembering the environment and for navigation. The first discovered spatial cell type was the hippocampal place cell, which fires selectively when the animal is at certain locations in its environment $[18,19]$. O'Keefe and his colleagues employed a technical development, the use of chronically implanted micro wires in freely moving animals to record the activity of single neurons [20].

More than 30 years later the grid cells were discovered in the medial entorhinal cortex [21]. These cells fire at multiple discrete and regularly spaced locations which form a hexagonal pattern [22]. Grid cells are supposed to provide an internal coordinate system, that is, to be part of the brain's metric for local space [23]. Place cells and grid cells were discovered in rats, but their existence was confirmed 
later in other species too, e.g. mice [24], monkeys [25, 26] and also humans $[27,28]$.

Each grid cell has a specific grid spacing, grid orientation and grid phase. These cells are arranged in discrete, relatively autonomous modules [29]. Each place is associated with a unique combination of active neurons, which collectively form a map of the local environment.

In the presubiculum and medial entorhinal cortex other spatial cell types exist also. The head direction cells fire only if the rat's head is pointing in a certain direction relative to external cues [30]. Border cells are activated when the animal is close to the wall of the recording enclosure or the edge of a platform [31]. Using combined optogenetic-electrophysiological technique it was demonstrated that grid cells, head direction cells and border cells have projections to the hippocampus and are involved in place field formation [32] [33].

In hippocampal place cells phase precession, a progressive advance of spike timing relative to the EEG theta phase, was observed when rats run through the place field [34]. A consequence of the phase shift is that, when the rat passes through a sequence of place fields, portions of this sequence will be replicated, in compressed form, within individual theta cycles. In successive theta cycles, cells representing overlapping place fields shift together in time and sustain a temporally ordered relationship with each other, so that the cells that fires on the earliest phase represents a place field whose center the animal traverses first. This compression and repetition of neural activity sequences makes possible to use long-term potentiation, which is limited to periods no longer than $50-100 \mathrm{~ms}$, to memorize the temporal structure of the rat's experience [35].

A discrete group of neurons that fire together form a cell assembly that represents a distinct cognitive entity. Cell assemblies activated when the animal occupies a particular location during awake behavior exhibit an increased tendency to fire together during subsequent sleep [36]. Reexpression during sleep of memory traces encoded during active behavior is important in memory consolidation.

Firing of some place cells is indicating not only the current location of the animal, but also might be informative about other locations that the animal cares about, such as the origin of the journey (retrospective coding) or the set of locations defining a further route (prospective coding) [37]. Cell assemblies carrying simultaneously information about the recent past, the present, and the imminent future represent a possible mechanism for episodic memory formation [38].

When rats pause at the choice point of a decision making task, hippocampal ensembles pre-play forward-directed paths corresponding to possible future actions, suggesting a mechanism for deliberation between concurrently available choices [39]. Possible future locations are probably retrieved from stored representations [23, 40].

The discoveries of John O'Keefe, May-Britt Moser and Edvard I. Moser were seminal in understanding how high level mental functions, far from primary sensory input and motor output, are represented in the brain, and how ensembles of specialized cells work together to compute complex cognitive functions and behaviour.

\section{References}

1. "The Nobel Prize in Physiology or Medicine 2014". Nobelprize.org. Nobel Media AB 2014. Web. 3 Nov 2014. <http://www.nobelprize.org/nobel_ prizes/medicine/laureates/2014/>

2. Nobel Lectures, Physiology or Medicine 1901-1921, Elsevier Publishing Company, Amsterdam, 1967

3. "The Nobel Prize in Physiology or Medicine 1904". Nobelprize.org. Nobel Media AB 2014. Web. 3 Nov 2014. <http://www.nobelprize.org/nobel_ prizes/medicine/laureates/1904/>

4. "The Nobel Prize in Physiology or Medicine 1906". Nobelprize.org. Nobel Media AB 2014. Web. 3 Nov 2014. <http://www.nobelprize.org/nobel_ prizes/medicine/laureates/1906/>

5. Presentation Speech by Professor G. Liljestrand, member of the Staff of Professors of the Royal Caroline Institute, on December 10, 1932, Nobel Lectures, Physiology or Medicine 1922-1941, Elsevier Publishing Company, Amsterdam, 1965

6. "The Nobel Prize in Physiology or Medicine 1932". Nobelprize.org. Nobel Media AB 2014. Web. 4 Nov 2014. <http://www.nobelprize.org/nobel_ prizes/medicine/laureates/1932/>

7. "The Nobel Prize in Physiology or Medicine 1936". Nobelprize.org. Nobel Media AB 2014. Web. 4 Nov 2014. <http://www.nobelprize.org/nobel_ prizes/medicine/laureates/1936/>

8. "The Nobel Prize in Physiology or Medicine 1944". Nobelprize.org. Nobel Media AB 2014. Web. 5 Nov 2014. <http://www.nobelprize.org/nobel_ prizes/medicine/laureates/1944/>

9. Presentation Speech by Professor R. Granit, Head of the Department of Neurophysiology of the Nobel Institute of the Royal Caroline Institute, "Physiology or Medicine 1944 - Presentation Speech". Nobelprize.org. Nobel Media AB 2014. Web. 4 Nov 2014. <http://www.nobelprize.org/ nobel_prizes/medicine/laureates/1944/press.html>

10. "The Nobel Prize in Physiology or Medicine 1963". Nobelprize.org. Nobel Media AB 2014. Web. 4 Nov 2014. <http://www.nobelprize.org/nobel_ prizes/medicine/laureates/1963/>

11. "The Nobel Prize in Physiology or Medicine 1970". Nobelprize.org. Nobel Media AB 2014. Web. 5 Nov 2014. <http://www.nobelprize.org/nobel_ prizes/medicine/laureates/1970/>

12. "The Nobel Prize in Physiology or Medicine 1981". Nobelprize.org. Nobel Media AB 2014. Web. 5 Nov 2014. <http://www.nobelprize.org/nobel_ prizes/medicine/laureates/1981/>

13. Presentation Speech by Professor David Ottoson of the Karolinska Institute, Nobel Lectures, Physiology or Medicine 1981-1990, Editor-inCharge Tore Frängsmyr, Editor Jan Lindsten, World Scientific Publishing Co., Singapore, 1993

14. The Nobel Prize in Physiology or Medicine 1991". Nobelprize.org. Nobel Media AB 2014. Web. 7 Nov 2014. <http://www.nobelprize.org/nobel_ prizes/medicine/laureates/1991/>

15. "The Nobel Prize in Physiology or Medicine 2000". Nobelprize.org. Nobel Media AB 2014. Web. 7 Nov 2014. <http://www.nobelprize.org/nobel_ prizes/medicine/laureates/2000/>

16. Moser El, Roudi Y, Witter MP et al. Grid cells and cortical representation. Nature Reviews Neuroscience. 2014; 15(7):466-481.

17. Felleman DJ, Van Essen DC. Distributed Hierarchical Processing in the Primate Cerebral Cortex. Cerebral Cortex. 1991; 1(1):1-47.

18. O'Keefe J, Dostrovsky J. The hippocampus as a spatial map. Preliminary evidence from unit activity in the freely-moving rat. Brain Res. 1971; 34(1):171-175.

19. O'Keefe J. Place units in the hippocampus of the freely moving rat. Exp Neurol. 1976; 51(1):78-109.

20. Strumwasser F. Long-term recording' from single neurons in brain of unrestrained mammals. Science. 1958; 127(3296):469-470.

21. Fyhn M, Molden S, Witter MP et al. Spatial representation in the entorhinal cortex. Science. 2004; 305(5688):1258-1264.

22. Hafting T, Fyhn M, Molden S et al. Microstructure of a spatial map in the entorhinal cortex. Nature. 2005; 436(7052):801-806.

23. Moser El, Kropff E, Moser MB. Place cells, grid cells, and the brain's spatial representation system. Annu Rev Neurosci. 2008; 31:69-89.

24. Fyhn M, Hafting T, Witter MP et al. Grid cells in mice. Hippocampus. 2008; 18(12):1230-1238. 
25. Ono T, Nakamura K, Nishijo H, Eifuku S. Monkey hippocampal neurons related to spatial and nonspatial functions. J Neurophysiol. 1993; 70(4):1516-1529.

26. Rolls ET, Robertson RG, Georges-Francois P. Spatial view cells in the primate hippocampus. European Journal of Neuroscience. 1997; 9(8):1789-1794.

27. Ekstrom AD, Kahana MJ, Caplan JB et al. Cellular networks underlying human spatial navigation. Nature. 2003; 425(6954):184-188.

28. Jacobs J, Weidemann CT, Miller JF et al. Direct recordings of gridlike neuronal activity in human spatial navigation. Nat Neurosci. 2013; 16(9):1188-1190.

29. Stensola H, Stensola T, Solstad $T$ et al. The entorhinal grid map is discretized. Nature. 2012; 492(7427):72-78.

30. Taube JS, Muller RU, Ranck JB, Jr. Head-direction cells recorded from the postsubiculum in freely moving rats. I. Description and quantitative analysis. J Neurosci. 1990; 10(2):420-435.

31. Solstad T, Boccara CN, Kropff E et al. Representation of geometric borders in the entorhinal cortex. Science. 2008; 322(5909):1865-1868.

32.Zhang SJ, Ye J, Miao C et al. Optogenetic dissection of entorhinal- hippocampal functional connectivity. Science. 2013; 340(6128):1232627.

33. Bush D, Barry C, Burgess N. What do grid cells contribute to place cell firing? Trends Neurosci. 2014; 37(3):136-145.

34. O'Keefe J, Recce ML. Phase relationship between hippocampal place units and the EEG theta rhythm. Hippocampus. 1993; 3(3):317-330.

35. Skaggs WE, McNaughton BL, Wilson MA, Barnes CA. Theta phase precession in hippocampal neuronal populations and the compression of temporal sequences. Hippocampus. 1996; 6(2):149-172.

36. Wilson MA, McNaughton BL. Reactivation of hippocampal ensemble memories during sleep. Science. 1994; 265(5172):676-679.

37. Ferbinteanu J, Shapiro ML. Prospective and retrospective memory coding in the hippocampus. Neuron. 2003; 40(6):1227-1239.

38. Buzsaki G. Neural syntax: cell assemblies, synapsembles, and readers. Neuron. 2010; 68(3):362-385.

39. Johnson A, Redish AD. Neural ensembles in CA3 transiently encode paths forward of the animal at a decision point. J Neurosci. 2007; 27(45):1217612189.

40. Pfeiffer BE, Foster DJ. Hippocampal place-cell sequences depict future paths to remembered goals. Nature. 2013; 497(7447):74-79. 